\title{
MODERN EXPERIENCE IN DYNAMIC STATES SIMULATION OF PIPELINE SYSTEMS AND THE POSSIBILITY OF ITS APPLICATION TO SOLVING IDENTIFICATION PROBLEMS
}

\author{
Oksana A. Grebneva ${ }^{1, *}$, and Nikolay N. Novitsky ${ }^{1}$ \\ ${ }^{1}$ Melentiev Energy Systems Institute of Siberian branch of Russian Academy of Science (ESI SB \\ RAS), department of pipeline and hydraulic systems, Lermontov Street 130, Irkutsk, Russia, 664033
}

\begin{abstract}
The effectiveness of solving the problems of development, reconstruction and control of pipeline systems is directly related to the level of application of methods for mathematical modeling and computer technology, which cannot be provided in isolation from the problems of reliability of information about their actual characteristics and parameters, as well as the degree of adequacy of used models. These problems constitute the content of identification tasks. The trend of transition to intelligent pipeline systems requires consideration of their control processes in real time, and hence the consideration of identification problems as passive identification problems based on the results of dynamic measurement data. The article provides an analysis of the existing experience in the field of dynamic states modeling of pipeline systems of various types and purposes, as well as mathematical formulations of the identification problem depending on the degree of parametric and state nonstationarity of systems, on the method and rate of data acquisition.
\end{abstract}

\section{Introduction}

A large number of works both in Russia [1-6] and abroad [7-9] are devoted to the identification of pipeline systems (PLSs) of various types and purposes, which indicates their relevance, complexity and multidimensionality.

Recently, in connection with new trends in the transition to intelligent PLS, it is necessary to consider the processes of their control in real time. This, in turn, requires the consideration of the identification problems as passive identification problems based on the results of dynamic (obtained during the operation of the system and changing in time) measurement data.

The subject of the article is the analysis of the existing experience in the field of modeling the dynamic states of PLSs of various types and purposes for the possibility of its subsequent use in solving identification problems.

*Corresponding author: oksana@,isem.irk.ru 


\section{The problem of dynamic identification of PLS}

As mentioned above, the purpose of identification is to obtain the parameters and characteristics of PLS with the required accuracy (ensuring the required adequacy of the used mathematical models), which undergo significant changes during operation, and the coefficients of these characteristics are not available for direct measurement or observation. In the previously developed identification methods [10], it was assumed that the coefficients of characteristics were set expertly with some initial approximation. When proceeding to the consideration of dynamic identification, it is necessary to take into account the change in these characteristics, as well as operation states of PLSs over time. In this case, the following tasks arise: 1) estimation of the state according to the measurement results; 2) parametric identification; 3) forecasting.

Technological transformation and modernization of PLSs due to the intensive introduction of new equipment, including measuring instruments, systems for acquisition and processing of measurement data, makes it possible to obtain a selection based on measurement data over time for various states of their operation. Nevertheless, the existing level of equipping with measuring instruments, which, as a rule, are installed at the main object (Heat Sources, Pumping Station, etc.) for technological and commercial accounting, is not always sufficient to solve identification problems. However, this ensures the availability of a basic composition of measurements, changing, which, it can provide the required quality of identification. These circumstances, in turn, make relevant a special class of problems and methods of current (operational) identification, involving the refinement of previously obtained results on the basis data received without using retrospective measurement information. In this case, instead of the task of synthesis of information-measuring systems, the task of their development arises, providing the necessary conditions for the formulation and solution of dynamic identification problems, as well as the development and application of new methods that provide the ability to process large volume of measured information at the rate of its arrival. The methodological basis for solving the problems of development of information-measuring systems can be developed earlier in the framework of the active identification methodology [11], the method of optimization of the measurements composition [12].

The transition to dynamic identification methods will make it possible to lower the requirements for the composition and number of measuring instruments due to an increase in the degree of overdetermination of the problem due to additional information obtained in the form of dynamic equations.

\section{Modeling of PLS operating states}

Consideration of PLS control processes in real time is reduced to the consideration of two types of states of their functioning: 1) steady-state (stationary); 2) dynamic (nonstationary).

Stationary states of PLSs operation are characterized by a change in parameters only in spatial coordinates, for example, along the length of pipelines. Therefore, when modeling them, all parameters are considered as constant values for a specific period of time. In real conditions, such states are short-term, and their models can be effectively used only when solving design and reconstruction problems, as well as when planning the conditions for conducting special tests (active identification). However, when solving control problems, instead of stationary states, it is necessary to consider dynamic (time-varying) states, which are basic in the operation of PLSs.

A feature of non-stationary states is the change in parameters not only in spatial coordinates, but also in time. Depending on the inertial properties of a specific PLS and the 
nature of the disturbing influences, non-stationary states can arise with natural nonstationarity of consumption processes (quasi-stationary states [13-15]) and with violations of the normal state of PLSs operation due to emergencies accompanied by the occurrence of unacceptable pressures under the conditions of equipment strength (transient states water hammer [16-22]). The latter, as well as stationary states, are short-term processes and are not typical for the functioning of PLS. Most of the time, these systems operate in states smoothly varying in time (quasi-stationary regimes), which are monotonic and caused by long-term disturbances.

When solving any problem in the process of PLS controlling, it is necessary to give their mathematical description. Depending on the type of the system operation state under consideration, this can be done using either algebraic (stationary states) or differential (nonstationary states) equations.

Stationary state models. When modeling the steady-state (stationary) states of PLSs operation, there are models of hydraulic [23-26], temperature [27] and thermohydraulic states of PLSs [10].

The model of the thermohydraulic state [10] (1)-(3) includes the equation of the first (material balance at the nodes) (1) and the second (2) Kirchhoff laws, as well as equation (3), which reflects the laws of pressure drop along the branches. The temperature state model (4)-(5) includes the heat balance equation at the nodes (4), the condition for the complete mixing of flows (5) in the nodes and the equation (6), which reflects the laws of temperature drop with the flow of the medium along the branches.

Non-stationary state models. This class of models has long been used to solve problems of analysis and simulation. Traditionally, the system of partial differential equations in [16] is used to model unsteady fluid flow states. The first equation of the system is a modification of the motion equation, the second is a modified continuity equation of the flow.

The model presented in [16] describes the phenomenon of water hammer, which, as mentioned above, occurs when the normal state of PLS operation is disturbed, has a shortterm character and is not usual for functioning states of PLSs. The use of this model for describing non-stationary states of real dimensionality pipelines increases the complexity and laboriousness of calculations. Therefore, it is not advisable to use this model to describe smoothly varying in time (quasi-stationary) states. The transition to the consideration of models of quasi-stationary states of PLSs is due to the need to take into account the following factors of nonstationarity:

1. The presence of accumulating tanks, for example, in water supply systems: pressure tanks, counter tanks, reservoirs at the inlet of pumping stations to smooth out their operating states; in heat supply systems: accumulating tanks of heated water to provide hot water for consumers during maximum load hours, etc. The authors in [28] propose a system of equations in differential form for modelling the reservoir level in a non-stationary state of water supply system.

2. Transport lag of quality parameters of the supplied medium (temperature, physical and chemical properties, etc.), which propagate with the speed of the medium. In [29], an equation for the temperature dynamics is proposed in terms of the average temperature lag coefficient.

In many works [13-15], when modeling non-stationary operation states of PLSs, the authors attempt to reduce these states to a set of continuously following one after another stationary states in which the parameters characterizing the system aren't changed during the observation time. 


\section{Statement of the dynamic identification problem}

Reviewed the issues of PLSs modeling, it gives to proceed to the mathematical formulation of the identification problem depending on the degree of parametric and state nonstationarity, as well as on the method and rate of data acquisition [4-5]:

1. Retrospective identification consists in determining of estimates for the vector of elements parameters $(\boldsymbol{\alpha})$ by the results of measuring of parameters $N$ steady states: $\tilde{\mathbf{R}}_{1}^{(u)}, u=\overline{1, N}$, at that, $\alpha=$ const for the period of measurements. Under these conditions, the sequence of states in time does not matter. The processing of measurement results can be carried out autonomously within the framework of the basic mathematical formulation with criterion (1) under constraints in the form of flow distribution $\overline{\mathrm{U}}(\mathbf{Z})=\overline{\mathrm{U}}\left(\mathbf{Z}_{\mathbf{1}}, \mathbf{Z}_{\mathbf{2}}\right)=\mathbf{0}$, where $\overline{\mathrm{U}}(\mathbf{Z})=\left\{\mathrm{U}\left(\mathbf{R}^{(1)}, \boldsymbol{\alpha}\right), \mathrm{U}\left(\mathbf{R}^{(2)}, \boldsymbol{\alpha}\right), \ldots, \mathrm{U}\left(\mathbf{R}^{(N)}, \boldsymbol{\alpha}\right)\right\}$ и $\mathbf{Z}=\left\{\mathbf{R}^{(1)}, \mathbf{R}^{(2)}, \cdots, \mathbf{R}^{(N)}, \boldsymbol{\alpha}\right\}$.

$$
\Phi\left(\mathbf{Z}_{1}\right)=\frac{1}{2}\left(\tilde{\mathbf{Z}}_{1}-\mathbf{Z}_{1}\right)^{T} \mathbf{C}_{\mathrm{Z1}}^{-1}\left(\tilde{\mathbf{Z}}_{1}-\mathbf{Z}_{1}\right)=\frac{1}{2} \mathbf{e}^{T}\left(\mathbf{Z}_{1}\right) \mathbf{C}_{\mathrm{Z} 1}^{-1} \mathbf{e}\left(\mathbf{Z}_{1}\right),
$$

where $\mathbf{Z}_{1}$ - vector of measurements for model parameters; $\mathbf{C}_{\mathbf{Z}_{1}}$ - covariance matrix of measurement error $\xi_{\mathbf{Z}_{1}}=\tilde{\mathbf{Z}}_{1}-\breve{\mathbf{Z}}_{1}$ is assumed to be given; $\tilde{\mathbf{Z}}_{1}, \breve{\mathbf{Z}}_{1}-$ vectors of measured and true values $\mathbf{Z}_{1}$.

The presence of permanently operating systems for acquisition of measurement information imposes special requirements on identification methods and algorithms, since calculations must be synchronized with the rate of receipt of measurements, and storage and simultaneous processing of data with $N \rightarrow \infty$ becomes impossible, whatever the resources of the used computer technology. These circumstances, in turn, make relevant a special class of problems and methods of current (operational) identification, involving the refinement of previously obtained results on the basis of newly received data without using retrospective measurement information. Despite the fact that the application of this scheme is supposed to be carried out in real time, it can be implemented on the basis of steady-state flow distribution models - by excluding from processing measurements corresponding to transient processes caused by discrete disturbances.

2. Identification of dynamic characteristics. In many cases, the condition $\boldsymbol{\alpha}=$ const cannot be taken as a basis. With the current identification carried out on an unlimited time interval, tracking changes in actual values can be one of their main goals. At the same time, considering $\boldsymbol{\alpha}$ as some unknown function of time means that each new state of PLS, for which measurements $\tilde{\mathbf{R}}_{1}(t)$ are used, introduces additional unknowns $\boldsymbol{\alpha}(t)$ and does not override the task. This is solution nevertheless turns out to be possible on the basis of additional a priori information on the behavior $\alpha$ in time. In this case, the following situations are possible:

a) the law of change $\boldsymbol{\alpha}$ is specified as an explicit deterministic function of time with a finite number of parameters

$$
\alpha_{i}(t)=\varphi_{i}\left(\beta_{i}, t\right), i=\overline{1, n},
$$

where $\varphi_{i}$ - dynamic characteristic of branche $i$ of network schema; $\beta_{i}-$ the vector of its parameters, which are no longer dependent on time. Substitution (2) into the initial equations of the flow distribution model $\mathrm{U}[\mathbf{R}(t), \boldsymbol{\alpha}(t)]=\mathbf{0}$ leads (replaced $\alpha$ by $\beta$ ) to the already considered current identification problem. The presence of the species $\phi_{i}\left(\beta_{i}, t\right)$ makes it possible to predict $\boldsymbol{\alpha}$ at an arbitrary point in time. The covariance matrix of prediction errors has the form $\overline{\mathbf{C}}_{\alpha}=(\partial \varphi / \partial \beta)^{T} \hat{\mathbf{C}}_{\beta}(\partial \varphi / \partial \beta)$, where $\hat{\mathbf{C}}_{\beta}$ - estimates 
covariance matrix $\hat{\beta} ; \partial \varphi / \partial \beta$ - partial differential matrix of a vector-function $\varphi(\boldsymbol{\beta}, t)$ в точке $\hat{\beta}, t$.

b) function (2) is unknown, but the nature of the behavior $\boldsymbol{\alpha}$ in time is known. As a rule, one can rely on the assumption of a directed and monotonic change $\boldsymbol{\alpha}(t)$. Expanding an unknown function $\alpha_{i}(t)$ in the surrounding area $t_{0}$ in the Taylor series, limited, for example, by members of the second order $\alpha_{i}\left(t_{0}+\Delta t\right)=\alpha_{i}\left(t_{0}\right)+\left(\partial \alpha_{i} / \partial t\right) \Delta t+\frac{1}{2}\left(\partial^{2} \alpha_{i} / \partial t^{2}\right) \Delta t^{2}$, where $\Delta \mathrm{t}=t-t_{0}$, and designating: $\beta_{i 0}=\alpha_{i}\left(t_{0}\right), \beta_{i 1}=\left(\partial \alpha_{i} / \partial t\right), \beta_{i 2}=\frac{1}{2}\left(\partial^{2} \alpha_{i} / \partial t^{2}\right)$, we have $\alpha_{i}(t)=\varphi_{i}\left(\beta_{i}, t\right)+\xi_{\alpha, i}(t)$, где $\varphi_{i}\left(\beta_{i}, t\right)=\beta_{i 0}+\beta_{i 1} \Delta t+\beta_{i 2}(\Delta t)^{2}, \xi_{\alpha, i}(t)$ - approximation error. If we neglect the value $\xi_{\alpha, i}(t)$, then the problem is reduced to the previous case.

c) law of change $\boldsymbol{\alpha}$ is given in the form of a stochastic function, which in general for the network can be represented in the form

$$
\boldsymbol{\alpha}(t)=\varphi(\boldsymbol{\beta}, t)+\boldsymbol{\xi}_{\alpha}(t) .
$$

In most cases, the vector $\xi_{\alpha}(t)$ (composed of values $\xi_{\alpha, i}(t), i=\overline{1, n}$ ) can be interpreted as noise with zero mean and unknown, but constant in time, covariance matrix $\mathbf{C}_{\alpha}$. Thus, when processing data for state $N$, received at the time $t$, there are errors of three types: $\xi_{R 1}(t)$ - error of measurements, $\xi_{\alpha}(t)$ - error of model (3), $\boldsymbol{\xi}_{\beta}(t)$ - error of identification $\boldsymbol{\beta}$ in the previous stages. Forming the joint density of their distribution, and passing to the loss function, we obtain the minimum problem $\Phi[\mathbf{R}(t), \boldsymbol{\alpha}(t), \boldsymbol{\beta}]=\frac{1}{2}\left(\mathbf{e}_{R 1}^{T} \mathbf{C}_{R 1}^{-1} \mathbf{e}_{R 1}+\mathbf{e}_{\alpha}^{T} \tilde{\mathbf{C}}_{\alpha}^{-1} \mathbf{e}_{\alpha}+\mathbf{e}_{\beta}^{T} \mathbf{C}_{\beta}^{-1} \mathbf{e}_{\beta}\right) \quad$ with restrictions $\mathrm{U}[\mathbf{R}(t), \boldsymbol{\alpha}(t)]=0$, where $\quad \mathbf{e}_{R 1}=\mathbf{e}[\mathbf{R}(t)]=\tilde{\mathbf{R}}_{1}(t)-\mathbf{I}_{R 1} \mathbf{R}(t) ; \quad \mathbf{e}_{\alpha}=\mathbf{e}[\boldsymbol{\alpha}(t), \boldsymbol{\beta}]=\tilde{\boldsymbol{\alpha}}(t)-\varphi(\boldsymbol{\beta}, t) ; \quad \mathbf{e}_{\beta}=\mathbf{e}(\beta)=\tilde{\boldsymbol{\beta}}-\boldsymbol{\beta} ;$ $\tilde{\boldsymbol{a}}(t)$ - predicted value $\boldsymbol{\alpha}(t) ; \tilde{\mathbf{C}}_{\alpha}$ - posterior value $\mathbf{C}_{\alpha}$, after processing of the state $(N-1)$. The method of sequential estimation is also applicable in this case, assuming only the expansion of the composition of unknowns, and the covariance matrix of forecast errors $\boldsymbol{\alpha}(t)$ here has the form $\overline{\mathbf{C}}_{\alpha}=(\partial \varphi / \partial \beta)^{t} \hat{\mathbf{C}}_{\beta}(\partial \varphi / \partial \beta)+\tilde{\mathbf{C}}_{\alpha}$.

d) dependence $\boldsymbol{\alpha}(t)$ is random. Assuming that fluctuations $\boldsymbol{\alpha}(t)$ do not exceed on average the value itself $\boldsymbol{\alpha}(t)$, it can set the problem of estimating the mathematical expectation $\boldsymbol{\alpha}(t)$, which denote as $\boldsymbol{\beta}=\mathbf{E}[\boldsymbol{\alpha}(\mathrm{t})]$. In this case, we have the following model $\boldsymbol{\alpha}(t)=\boldsymbol{\beta}+\boldsymbol{\xi}_{\alpha}(t)$. Obviously, this situation is a limiting case (2).

For this case, the sequential estimation technique is also applicable, which assumes only the expansion of the composition of unknowns.

3. Adaptive identification. If the rate of arrival of measurements is high, it is possible to carry out the estimation without invoking dynamic characteristics, assuming that in the vicinity of the current moment of time the changes in the vector are insignificant (at least against the background of measurement noise), and this neighborhood itself is large to accumulate information about the parameters, which allow estimate with sufficient accuracy. The traditional approach to the construction of adaptive identification algorithms is to take into account the aging of the measurement information obtained outside the regularity interval. The corresponding modification of the objective function 
$\Phi\left(\mathbf{R}^{(1)}, \ldots, \mathbf{R}^{(N)}, \boldsymbol{\alpha}\right)=\frac{1}{2}\left[\sum_{u=1}^{N} \mathbf{d}^{(u)} \mathbf{e}^{t}\left(\mathbf{R}^{(u)}\right) \mathbf{C}_{R 1}^{-1} \mathbf{e}\left(\mathbf{R}^{(u)}\right)+\mathbf{e}^{T}(\boldsymbol{\alpha}) \mathbf{C}_{\alpha, 0}^{-1} \mathbf{e}(\boldsymbol{\alpha})\right]$, where $\quad d^{(u)} \quad-\quad$ scalar function of reliability of measurement $u$.

There are two most common variants of the specification for the reliability function $d^{(u)}:$ 1) sliding window method; 2) exponential weighting (smoothing) method.

4. Identification of dynamic states. The involvement (where it is really necessary) of the equations of unsteady flow distribution in the role of state models, although it leads to the complication of identification procedures, provides significant advantages, the main one of which is that the dimension of the vector of the estimated parameters does not increase with an increase in the number of measurements, due to the addition equations of dynamics.

Thus, discrete analogues of the equations of unsteady flow distribution, at least numerically, can be represented in the form of two main subsystems of equations: 1) dynamics model $\mathbf{X}_{\mathrm{R}}^{(u)}=\varpi^{(u)}\left(\mathbf{X}_{\mathrm{R}}^{(u-1)}, \boldsymbol{\alpha}\right) ; \quad$ 2) measurements model: $\tilde{\mathbf{Z}}_{1}^{(u)}=\psi^{(u)}\left(\mathbf{X}_{R}^{(u)}, \boldsymbol{\alpha}\right)+\xi_{Z 1}^{(u)}$, where index $u$ refers to a discrete moment in time $t^{(u)}$. The dynamics model allows the state $\mathbf{X}_{R}^{(u)}$ at any time $t^{(u)}$ represent as a function of the initial state $\quad \mathbf{X}_{R}^{(0)}: \quad \mathbf{X}_{\mathrm{R}}^{(u)}=\bar{\varpi}^{(u)}\left(\mathbf{X}_{\mathrm{R}}^{(0)}, \boldsymbol{\alpha}\right), u=\overline{1, N} . \quad$ By designating $\boldsymbol{\gamma}=\left[\left(\mathbf{X}_{R}^{(0)}\right)^{t}, \boldsymbol{\alpha}^{t}\right]^{t}, \quad$ get $\tilde{\mathbf{Z}}_{1}^{(u)}=\psi^{(u)}(\gamma)+\xi_{Z 1}^{(u)}, u=\overline{1, N}$.

Thus, the problem of parametric identification in the case under consideration can be reduced to solving the problem for an unconditional minimum $\Phi(\gamma)=\frac{1}{2} \sum_{u=1}^{N}\left[\tilde{\mathbf{Z}}_{1}^{(u)}-\psi^{(u)}(\gamma)\right]^{T} \mathbf{C}_{Z 1}^{-1}\left[\tilde{\mathbf{Z}}_{1}^{(u)}-\boldsymbol{\psi}^{(u)}(\gamma)\right]$

Although with growth $N$ the dimension of the equations system does not increase, the complexity of the problem significantly increases, since at each iteration it is necessary to calculate the entire trajectory of the dynamics of states $\left\{R_{k}^{(u)}, u=\overline{1, N}\right\}$, and also matrices of derivatives. Therefore, perhaps the only possible approach to identifying of PLS as dynamic objects in real time is to use various methods of decomposition problem. One of such methods is the transition from the problem of estimating the initial state to the problem of estimating the current state, without recalculating the dynamics of the process at the previous moments of time. This technique, which can be interpreted as applying the idea of sequential estimation to the estimation of parameters of dynamic models, is also known as the Kalman filter.

\section{Conclusion}

Within the framework of this work, a study of the problem of modeling of PLSs operation states was carried out. The analysis of the models existing in the modern researches for the description of dynamic states is carried out.

The structuring is given and the content of the main identification tasks is disclosed depending on the degree of parametric and state nonstationarity of PLSs, as well as on the method and rate of data acquisition.

In general, the results of this study create the basis for dynamic identification problems for various purposes, conditions and types of PLSs.

The research was carried out within the project III.17.4.3 of the Fundamental research program of SB RAS (AAAA-A17-117030310437-4) 


\section{References}

1. RD34.20.519-97 (Moscow, Service of Excellence, 1998) [in Russian]

2. RD34.20.519-97 (Moscow, Service of Excellence, 1998) [in Russian]

3. $R D$ 153.34.1-20.526-00 (Moscow, AOOT «VTI», 2001) [in Russian]

4. N.N. Novitsky, News of the Russian Academy of Sciences. Energy, 6, 87 (2000)

5. N.N. Novitsky, System studies of energy problems, 252 (Novosibirsk, Science, 2000) [in Russian]

6. N.N. Novitsky, Evaluation of hydraulic circuits parameters (Science, 1998) [in Russian]

7. Srinivasa Lingireddy, Lindell E. Ormsbee. Civil Engineerng and environmental Systems, 19(1), 13 (2012)

8. S. Alvisi, E. Creaco, M. Franchini, Urban Water Journal, 8(4), 203 (2011)

9. Guoping Yu., Roger S. Powell, International Journal of Systems Science, 25(12), 2155 (1994)

10. O.A. Grebneva, N.N. Novitsky. Thermal Engineering, 61 (10), 754 (2014)

11. O.A. Grebneva, N.N. Novitsky. Pipeline Energy Systems: Methodological and Applied Problems of Mathematical Modelling, 220 (Science, Novosibirsk, 2015) [in Russian]

12. O.A. Grebneva, N.N. Novitskii, Thermal Engineering, 61(9), 685 (2014)

13. L.P. Kanina, G.A. Chapkina, Proceedings of the All-Russian scientific seminar "MMMOAOSODPHS", 205 (Irkutsk, ISEM SB RAS, 2016)

14. A.D. Tevyashev, A.A. Mamedova, V.A. Frolov, Eastern European Journal of Advanced Technologies, 1 (7(43)), 30 (2010) [in Russian]

15. Ezio Todini. Journal of Hydro informatics, 9(13.2), 167 (2011)

16. E.N. Zhukovsky, About water hammer in pipes (Moscow, 1949) [in Russian]

17. I.A. Charny, Unsteady motion of a real liquid in pipes (Moscow, GITTL, 1961) [in Russian]

18. K.P. Vishnevsky, Intensification and optimization of urban and industrial water pipelines (Moscow, 1973) [in Russian]

19. B.N. Gromov, L.P. Kanina, et al., Teploenergetika, 7 (1981) [in Russian]

20. A.A. Atavin, V.V. Tarasevich, Hydraulic chains. Development of the theory and applications, 16 (Novosibirsk, Science, 2000) [in Russian]

21. V.V. Tarasevich, Izvestiya of higher educational institutions. Construction, 7 (691), 92 (2016) [in Russian]

22. R. Wang, Z. Wang, Th. M. Walski, et al., 11th International Conference on Computing and Control for Water Industry, 877 (2011)

23. A.P. Merenkov, V.Ya. Hasilev, Theory of hydraulic circuits (1985) [in Russian]

24. M.M. Andriyashev, Hydraulic calculations of water pipelines and water supply networks (Moscow, Stroyizdat, 1964) [in Russian]

25. V.G. Lobachev, Questions of rationalization of calculations of water supply networks (Moscow, ONTI, 1936)

26. H. Cross Urbana, Illinois: Eng. Exp. Station of Univ. of Illinois, 286 (1936)

27. E.V. Sennova, V.G. Sidler, Mathematical modeling and optimization of developing heat supply systems (Novosibirsk, Nauka, 1987)

28. J. Zheng, Zh. Zhou, J. Zhao, et al., Applied Thermal Engineering, 123, 682 (2017)

29. I. Gabrielaitiene, B. Bohm, B. Sunden, Energy Conversion and Management, 48, 78 (2007) 